\title{
Genetic modification of mesenchymal stem cells to enhance their anti-tumor efficacy
}

\author{
Swathi Vangala ${ }^{1}$, Gopikrishna Moku
}

Cite this article: Vangala S, Moku G: Genetic modification of mesenchymal stem cells to enhance their anti-tumor efficacy. Asia-Pac J Oncol 2021; 2: 1-6. https://doi.org/10.32948/ajo.2021.03.28

\begin{abstract}
Non-hematopoietic mesenchymal stem cells (MSCs) are widely used in regenerative medicine and tissue engineering as they possess multilineage differentiation potential and self-renewal properties. MSCs can be easily isolated from several tissues and expanded following standard cell culture procedures. MSCs have the capability of mobilization to the tumor site; so, they can automatically relocate to the tumor sites through their chemokine receptors following intravenous transplantation. In this respect, they can be used for MSC-based gene therapy. In this therapeutic technique, beneficial genes are inserted by viral and non-viral methods into MSCs that lead to transgene expression in them. Genetic modifications of MSCs have been widely studied and thoroughly investigated to further enhance their therapeutic efficacy. The current strategies of MSC-based therapies emphasize the incorporation of beneficial genes, which will enhance the therapeutic ability of MSCs and have better homing efficiency. Non-viral methods produce less toxicity and immunogenicity compared to viral gene delivery methods and thus represent a promising and efficient tool for the genetic engineering of MSCs. Several non-viral gene delivery strategies have been developed in recent decades, and some of them have been used for MSCs modification. This mini review provides an overview of current gene delivery approaches used for the genetic modification of MSCs with beneficial genes including viral and non-viral vectors.
\end{abstract}

Key words Mesenchymal stem cells (MSC), cell-based therapy, genetic modification, viral and non-viral vectors, transfection, gene therapy

1. Telangana Social Welfare Residential Degree College for Women, Bhupalapally 506 169, Telangana, India.

2. Department of Physical Sciences, Kakatiya Institute of Technology and Science, Yerragattu Gutta, Warangal 506 015, Telangana, India.

Correspondence: Gopikrishna Moku (Department of Physical Sciences, Kakatiya Institute of Technology and Science, Yerragattu Gutta, Warangal 506 015, Telangana, India; E-mail: gopikrishna.moku@gmail.com). 


\section{Introduction}

MSCs are multipotent adult stromal cells that can be isolated from different sources, including amniotic fluid, bone marrow, dental pulp, adipose tissue, and umbilical cord [1-5]. MSCs are classified as cells that can adhere to plastic, express the surface molecules CD73, CD90, and CD105 (approximately 95\% positive), but lack the expression of CD45, CD19, CD14, CD34, CD11b, CD79alpha, and HLA-DR. MSCs possess specific characteristics of stem cells and differentiate into chondroblasts, adipocytes, osteoblasts, myoblasts, fibroblasts, and cardiomyoblasts under in vitro differentiation conditions [6].

Several studies have been reported on the possible therapeutic mechanisms of MSCs for the regenerative treatment of untreatable diseases. (i) The homing property of MSCs, enable them to adhere to tumor sites [7]. The homing effect of MSCs theoretically implies that, in clinical applications, MSCs can be migrated to the tumor site using only intravascular transplantation of MSCs and not surgery. (ii) Although the ratio of differentiated cells to transplanted cells has been reported to be very low, MSCs can differentiate directly into damaged cells, facilitating repair [8, 9]. (iii) MSCs can control immune responses [10-12] and, by controlling the activity of immune cells [13-15], can facilitate the regeneration of damaged tissues. (iv) It has been stated that MSCs can express different trophic factors that can inhibit immune cell function, delay cell death at damaged sites by inhibitors, and promote progenitor/stem cell proliferation and target cell differentiation [10]. (v) It is recognized that MSCs are hypoimmunogenic or immune-privileged, enabling allogeneic MSC transplantation through major barriers to histocompatibility and the development of off-the-shelf therapies consisting of MSCs grown in culture [16].

More than 1,100 clinical trials using MSCs have been reported for different diseases (https://clinicaltrials.gov) based on the therapeutic potential of MSCs. However, MSCs based therapy has not yet demonstrated adequate therapeutic effects in humans, despite several clinical trials using MSCs. Thus, MSC priming [17, 18], genetic modification [19], Three-Dimensional (3-D) culture [20], and MSC-derived exosomes [21, 22] have been studied to enhance the therapeutic potential of MSCs. Upon delivery to the damaged site, and after exposure to inflammatory cytokines, MSCs release various factors that control the function of inflammatory cells; this is followed by treatment of the damage. Consequently, it is possible to enhance the therapeutic effect of MSCs by pre-exposing them to inflammatory cytokines such as IFN-g, TNF-a and IL-1b [18]. Three-dimensional stem cell culture using different scaffolds has been reported to enhance the efficiency of stem cell proliferation and differentiation [20] and increase their therapeutic effects in liver disease, peritonitis, kidney injury, and myocardial infection [23, 24]. Owing to the therapeutic effects of various trophic factors secreted by MSCs, their regenerative therapeutic effects can be improved by using the secretome or exosome extracted from MSC. As exosomes can be processed, qualitatively regulated and repeatedly administered, they are an ideal factor, which can be used for the treatment of acute diseases. Enhancing the expression of the target gene, which plays an important role in tissue regeneration, is one way to reliably boost the therapeutic impact of MSCs. Therefore, we will address gene delivery methods in MSCs in this study, which are known to have low transformation efficiencies, and discuss the development and therapeutic efficacy of functionally enhanced MSCs.

\section{Genetic modification of MSCs}

Since the MSCs are attracted to the affected and/or tumor site makes it possible to use MSCs as a vehicle for different therapeutic agents, including genes. There are several strategies for genetically modifying MSCs, but they can be broadly divided into methods that are viral and non-viral vector systems [25]. Viral transduction of MSCs is commonly achieved using lenti-, retro-, adeno- or adeno-associated virus without affecting their stem cell properties $[26,27]$. Non-viral methods of gene transfer encompass all physical and chemical methods of gene delivery. Non-viral techniques are effective because they are capable of transmitting larger transgenes than viral techniques, are more cost-effective and are ideal for production scale-up and trigger the less immune response. Single or mixed cationic lipids, peptides, surfactants, metals (gold, magnetic iron), polysaccharides, and synthetic polymers have been used in non-viral vector systems for genetic manipulation [28-30].

\section{Lentiviral vectors}

Lentiviruses have double-stranded RNA as their genetic material and can transduce both quiescent and dividing cells. After entering into the cells, they incorporate the genome of their vectors into the host genome, ensuring long-term transgenic expression [31]. These vectors are effective in converting dividing, non-dividing or slowdividing cells, without changing their viability and differentiation potential. Non-integrating lentivirus vectors have recently been produced by viral integrase alterations or long terminal repeats and have been used for stable and safe delivery of the gene, resulting in long-term transgene expression [32]. These are one of the most commonly used vectors in mesenchymal stem cellbased gene therapy and have advantages such as large genome size, stable gene transfer, and high infection efficiency [33]. Besides, lentiviruses can be transduced into non-dividing cells and last for various generations. MSCs engineered with lentiviral vectors overexpressing HSP70 enhanced survival and resistance to apoptosis under conditions of hypoxia and ischemia without causing the morphology, viability, or differentiation capabilities of MSCs [34]. Transduction of MSCs with PGC-1 $\alpha$ using lentiviral vectors reduced neuronal apoptosis and improved the capability of axonal regeneration in a rat model of traumatic spinal cord injury [35]. MSCs engineered with tumor necrosis factor (TNF)-related apoptosis-inducing ligand (TRAIL)-stimulated apoptosis in cancer cell lines, including colon, lung, pleural mesothelioma and oral squamous cancer [36]. However, the major concern with the use of lentiviral vectors is that they lack specificity, thus it can lead to the infection of cells that do not need to be transduced. Furthermore, the majority of lentiviruses that have been produced are derived from HIV, raising safety concerns for applications in in vivo gene therapy [37].

\section{Adenoviral (AV) vectors}

Adenoviruses (AVs) are non-enveloped viruses containing a double-stranded DNA genome with icosahedral nucleocapsids. Since they have a broad host range and can infect both dividing and non-dividing cells, adenoviruses are the most widely used gene delivery vector [38]. Non-pathogenicity is a significant advantage in their use as vectors for gene transfer. There is no risk of insertional mutagenesis and the payload capacity of these vectors in MSCs is high ( $\sim 36 \mathrm{~Kb})$. The efficacy of adenovirus gene transmission is closely related to the expression of Coxsackievirus and Adenovirus Receptors (CARs) on target cells [39]. Due to a very low expression level of CARs on MSCs [40], gene delivery efficacy using these vectors is very low. To enhance the efficacy of gene delivery by these vectors into MSCs, a capsid- and a fiberaltered adenovirus has been developed [41, 42]. Moreover, in bone marrow-derived MSCs, the initial robust expression of the newly inserted gene gradually declines after 21 days; this strategy could 
Table 1. Viral vectors used for the genetic modification of mesenchymal stem cells.

\begin{tabular}{lll} 
Viral vector Structure $\quad$ Advantages & Disadvantages \\
\hline
\end{tabular}

$\begin{array}{lll} & \text { Easy manipulation and production; } & \\ & \text { Easily cross nuclear membrane; } & \text { Can generate insertional } \\ \text { Lentiviral vectors ds RNA } & \text { infects dividing and quiescent cells; } & \\ & \text { replication incompetent; } & \\ & \text { no insertion into oncogene } & \text { Transient gene expression; } \\ & \text { High production of viral particles; } & \text { immunogenic; } \\ \text { Adenoviral vectors ds DNA } & \text { lower risk of genotoxicity; } & \text { insertional mutagenesis }\end{array}$

Easy manipulation and production;

Retroviral vectors ss RNA DNA incorporated into host cell genome;

Insertional mutagenesis;

can't cross nuclear membrane

long-term stable gene expression

Infects dividing and quiescent cells;

long-term gene expression;

Adeno-associated
virus-based vectors

(ong

Small DNA inserts

non-cytotoxic;

non-immunogenic

therefore only be extended to the transient expression of target genes [43]. Genetical modification of MSCs with bi-cistronic adenoviral vector encoding FGF2 and PDGF-BB has been shown to initiate collateral vessel formation and angiogenesis in a hind limb ischemia model [44]. Human placenta-derived Transduction of MSCs derived from human placenta with adenoviral vector expressing NK4, injected through the tail vein, delayed the growth of lung metastases in C-26 lung metastasis model [45]. However, adenoviruses have high immunogenicity, which restricts their use in gene therapy. CD4+, CD8+ and antigen presenting cells are triggered by direct administration of Adenoviral vectors with a transgene into the host cells. This activation of the immune response triggered by both transgenic and viral capsid protein expression can often lead to the removal of the viral particle and the silencing of the transgenes [46].

\section{Retroviral vectors}

Retroviruses are viruses of single-stranded RNA that have a lipid envelope and reverse transcriptase with receptor-binding proteins.
After binding to the receptor, the outside layer of the viral envelope integrates with the cellular membrane, internalizes the virus and releases the content into the cytoplasm. The viral RNA reversetranscribed to DNA utilizing reverse transcriptase and integrates into the host genome [47]. There are many difficulties in using Retroviral vectors for gene therapy, despite their high tropism to host cells, such as the absence of long-term transgene expression, ineffective transduction of MSCs, induction of insertional mutagenesis, and the requirement for high vector loads to be administered in several rounds to transduce host cells [39, 42].

\section{Adeno-associated virus-based vectors}

Adeno-associated viruses (AAVs) are small, single-stranded, non-pathogenic DNA viruses that are adenovirus-dependent for replication [48]. For the following reasons, AAVs are considered to be an attractive gene therapy vector: (i) despite their ubiquity in the human population, they have no association with any disease; (ii) many human tissues can be easily infected with these vectors; (iii) AAV vectors are not capable of replication without a helper 
adenovirus; (iv) these vectors can exist in an episomal form for long-term transgene expression; (v) nontoxic in clinical trials in humans [49]. Despite the significant advantages such as sitespecific integration, low immunogenicity the application of AAVs is restricted due to various reasons. Although AAVs can infect a broad spectrum of cells, they show certain serotype specificity towards the cell type being used [50]. One of the major obstacles in the clinical use of AAV is the presence of antibodies to AAV in the majority of the human population, which limits vector efficiency [51]. Such immune reactions were found to be more prevalent against AAV2 in particular. Another impediment is the need for the conversion of single-stranded DNA into double-stranded DNA before integration into the genome, which is a rate-limiting step. Therefore, the molecular details of the AAV and host interaction biology need to be thoroughly investigated and develop strategies develop to resolve the limitations and make use of the benefits provided by AAVs [39].

\section{Non-viral vectors}

Different non-viral (physical and chemical) methods are widely used to introduce therapeutic genes in to MSCs. Restricted immunogenicity, improved biosafety and high loading capability are the key advantages of using non-viral vectors. Various physical methods such as electroporation [52], nucleofection [53], sonotransfection [54] and nanoparticles [55] are extensively used to transduce MSCs with target genes. However, the application of non-viral systems in gene therapy has been limited because of their low transfection efficiency and transient transgene expression [56]. In addition, transfection reagents and/or procedures can enhance the cytotoxicity of MSCs, leading to cell death or senescence. Recently, Helledie et al. shown that electroporation is a superior lipofection gene delivery method in MSCs without instigating proliferation and differentiation potential loss, whereas lipofection with Lipofectamine2000 decreased proliferation rate and increased MSC cell death [57]. Compared to most viral methods, they identified a simple and effective electroporation protocol that resulted in transfection efficiency of up to $90 \%$, but the absolute transfection efficacy was approximately 35 percent. In another study, a novel method based on Therapeutic Ultrasound (TUS) has been developed for efficient gene delivery into MSCs [58]. MSCs were transfected using low intensity and moderate frequency TUS with plasmids encoding hemopexin-like domain fragment (PEX), an angiogenesis inhibitor. TUS-mediated PEX transfected MSCs expressed biologically active PEX without loss of stem and homing abilities and subsequently inhibited $70 \%$ of prostate tumor development in a mouse model [58]. Among conventional nonviral delivery systems, liposomes and polymers have been shown to transfect MSCs transiently [59-62]. Minicircles are tiny DNAbased vectors that are normally present in traditional plasmid vectors without prokaryotic backbone sequences. Minicircles have shown less toxicity and immunogenicity, higher transfection efficiency and longer transgene expression, in vitro and in vivo, compared to conventional plasmid vectors [63-65]. Several studies indicate that MSCs $[66,67]$ can be effectively and stably transfected by minicircles.

\section{Conclusions}

MSCs possess strong regenerative, pro-inflammatory, antiinflammatory and drug delivery properties. These features introduce MSCs as attractive cell-based therapeutics for inflammatory based medical conditions. The homing property of MSCs to the affected and/or tumor site makes it possible to use MSCs to deliver various therapeutic agents, including genes. MSCs engineered with the therapeutic agents not only have important therapeutic effects but also act predominantly in only the damaged site, reducing the expected frequency of side effects resulting from the non-selective action of the drug. Viral and nonviral vectors have been studied for gene delivery into MSCs, and MSCs equipped with genes have been reported to enhance their therapeutic effects significantly in the treatment of regenerative medicine and cancer. Viral vectors have drawbacks, such as high immunogenicity and insertional mutagenesis, but they have the benefits of the high efficiency of transfection and longterm expression of genes. In comparison, non-viral vector gene delivery has poor transfection efficiency and transient target gene expression. Therefore, to suit the therapeutic purpose, various types of vectors must be used depending on the disease being treated. In addition, to make use of the benefits of each vector and to compensate for the disadvantages, new methods must be created. In the future, if such research is carried out, it is expected that not only will the therapeutic impact of MSCs be improved, but the application of MSCs to different diseases will dramatically improve patients' quality of life.

\section{Ethics approval and consent to participate}

Not applicable.

\section{Funding}

Not applicable.

\section{Author contributions}

SW wrote the paper, GKM corrected the manuscript.

\section{Competing interests}

All authors disclose no competing interests.

\section{References}

1. Kim HJ, \& Park JS. Usage of human mesenchymal stem cells in cell-based therapy: Advantages and disadvantages. Development \& Reproduction 2017; 21: 1-10.

2. Hass R, Kasper C, Böhm S, Jacobs, R. Different populations and sources of human mesenchymal stem cells (MSC): A comparison of adult and neonatal tissue-derived MSC. Cell Communication and Signaling 2011; 9: 12.

3. Ocansey DKW, Pei B, Yan Y, Hui Q, Xu Z, Wenrong Xu, Fei $M$. Improved therapeutics of modified mesenchymal stem cells: an update. J Transl Med 2000; 18: 42.

4. Ankrum JA, Ong JF, Karp JM. Mesenchymal stem cells: Immune evasive, not immune privileged. Nat Biotechnol 2014; 32: 252-260.

5. Lalu MM, McIntyre L, Pugliese C, Fergusson D, Winston BW, Marshall JC et al. Safety of cell therapy with mesenchymal stromal cells (SafeCell): A systematic review and meta-analysis of clinical trials. PLoS One 2012; 7: e47559.

6. Shahror RA, Wu C-C, Chiang Y-H, Chen K-Y. Genetically Modified Mesenchymal Stem Cells: The Next Generation of Stem Cell-Based Therapy for TBI. Int J Mol Sci 2020; 21: 4051.

7. Fu X, Liu G, Halim A, Ju Y, Luo Q, Song G. Mesenchymal Stem Cell Migration and Tissue Repair. Cells 2019; 8: 784.

8. Xagorari A, Siotou E, Yiangou M, Tsolaki E, Bougiouklis D, et al. Protective effect of mesenchymal stem cell-conditioned medium on hepatic cell apoptosis after acute liver injury. Int J Clin Exp Pathol 2013; 6: 831-840

9. Yang YHK, Ogando CR, Wang See C, Tsui-Yun C, Gilda AB. Changes in phenotype and differentiation potential of human mesenchymal stem cells aging in vitro. Stem Cell Res Ther 2018; 
9: 131.

10. Ghannam S, Bouffi C, Djouad F, Jorgensen C, Noel D. Immunosuppression by mesenchymal stem cells: mechanisms and clinical applications. Stem Cell Res Ther 2010; 1(1): 2.

11. Sato K, Ozaki K, Oh I, Meguro A, Hatanaka K, et al. Nitric oxide plays a critical role in suppression of T-cell proliferation by mesenchymal stem cells. Blood 2007; 109: 228-234.

12. Spaggiari GM, Abdelrazik H, Becchetti F, Moretta L. MSCs inhibit monocyte-derived DC maturation and function by selectively interfering with the generation of immature DCs: central role of MSC-derived prostaglandin E2. Blood 2009; 113 :6576-6583.

13. Jo H, Eom YW, Kim HS, Park HJ, Kim HM, et al. Regulatory Dendritic Cells Induced by Mesenchymal Stem Cells Ameliorate Dextran Sodium Sulfate-Induced Chronic Colitis in Mice. Gut Liver 2018; 12: 664-673.

14. Nemeth K, Leelahavanichkul A, Yuen PS, Mayer B, Parmelee A, et al. Bone marrow stromal cells attenuate sepsis via prostaglandin $\mathrm{E}(2)$-dependent reprogramming of host macrophages to increase their interleukin-10 production. Nat Med 2009; 15: 42-49.

15. Park HJ, Kim J, Saima FT, Rhee KJ, Hwang S, et al. Adiposederived stem cells ameliorate colitis by suppression of inflammasome formation and regulation of M1-macrophage population through prostaglandin E2. Biochem Biophys Res Commun 2018; 498: 988995.

16. Wang D, Zhang H, Liang J, Li X, Feng X, et al. Allogeneic mesenchymal stem cell transplantation in severe and refractory systemic lupus erythematosus: 4 years of experience. Cell Transplant 2013; 22: 2267-2277.

17. Giuliani M, Bennaceur-Griscelli A, Nanbakhsh A, Oudrhiri N, Chouaib S, et al. TLR ligands stimulation protects MSC from NK killing. Stem Cells 2014; 32: 290-300.

18. Sharma RR, Pollock K, Hubel A, McKenna D. Mesenchymal stem or stromal cells: a review of clinical applications and manufacturing practices. Transfusion 2014; 54: 1418-1437.

19. Du Z, Wei C, Yan J, Han B, Zhang M, et al. Mesenchymal stem cells overexpressing C-X-C chemokine receptor type 4 improve early liver regeneration of small-for-size liver grafts. Liver Transpl 2013; 19: 215-225.

20. Arufe MC, De la Fuente A, Fuentes-Boquete I, De Toro FJ, Blanco FJ. Differentiation of synovial CD-105(+) human mesenchymal stem cells into chondrocyte-like cells through spheroid formation. J Cell Biochem 2009; 108: 145-155.

21. Chen TS, Lai RC, Lee MM, Choo AB, Lee CN, et al. Mesenchymal stem cell secretes microparticles enriched in pre-microRNAs. Nucleic Acids Res 2010; 38: 215-224.

22. Gnecchi M, He H, Liang OD, Melo LG, Morello F, et al. Paracrine action accounts for marked protection of ischemic heart by Aktmodified mesenchymal stem cells. Nat Med 2005; 11: 367-368.

23. Bartosh TJ, Ylostalo JH, Mohammadipoor A, Bazhanov N, Coble K, et al. Aggregation of human mesenchymal stromal cells (MSCs) into 3D spheroids enhances their antiinflammatory properties. Proc Natl Acad Sci USA 2010; 107: 13724-13729.

24. Wang CC, Chen CH, Hwang SM, Lin WW, Huang CH, et al. Spherically symmetric mesenchymal stromal cell bodies inherent with endogenous extracellular matrices for cellular cardiomyoplasty. Stem Cells 2009; 27: 724-732.

25. Marofi F, Vahedi G, Biglari A, Esmaeilzadeh A, Athari SS. Mesenchymal Stromal/Stem Cells: A New Era in the Cell-Based Targeted Gene Therapy of Cancer. Front Immunol 2017; 8: 1770.

26. Nowakowski A, Andrzejewska A, Janowski M, Walczak P, Lukomska B. Genetic engineering of stem cells for enhanced therapy. Acta Neurobiol Exp (Wars) 2013; 73: 1-18.

27. Oggu GS, Sasikumar S, Reddy N, Ella KKR, Rao CM, et al Gene Delivery Approaches for Mesenchymal Stem Cell Therapy: Strategies to Increase Efficiency and Specificity. Stem Cell Rev Rep 2017; 13: 725-740.
28. Foldvari M, Chen DW, Nafissi N, Calderon D, Narsineni L, et al. Non-viral gene therapy: Gains and challenges of non-invasive administration methods. J Control Release 2016; 240: 165-190.

29. Peer D, Karp JM, Hong S, Farokhzad OC, Margalit R, et al. Nanocarriers as an emerging platform for cancer therapy. Nat Nanotechnol 2007; 2: 751-760.

30. Tsutsui JM, Xie F, Porter RT. The use of microbubbles to target drug delivery. Cardiovasc Ultrasound 2004; 2: 23.

31. Naldini L, Blomer U, Gallay P, et al. In vivo gene delivery and stable transduction of nondividing cells by a lentiviral vector. Science 1996; 272: 263.

32. Shaw A, Cornetta K. Design and Potential of Non-Integrating Lentiviral Vectors. Biomedicines 2014; 2: 14-35.

33. Nayerossadat N, Maedeh T, Ali PA. Viral and nonviral delivery systems for gene delivery. Adv Biomed Res 2012; 1: 27.

34. Xiao X, Li J, Samulski RJ. Efficient long-term gene transfer into muscle tissue of immunocompetent mice by adeno-associated virus vector. J Virol 1996; 70: 8098-8108.

35. Hu J, Lang Y, Zhang $\mathrm{T}$, et al. Lentivirus-mediated PGC-1 $\alpha$ overexpression protects against traumatic spinal cord injury in rats. Neuroscience 2016; 328: 40-49.

36. Yuan ZQ, Kolluri KK, Sage EK, Gowers KH, Janes SM. Mesenchymal stromal cell delivery of full-length tumor necrosis factor-related apoptosis-inducing ligand is superior to soluble type for cancer therapy. Cytotherapy 2015; 17: 885-896.

37. Tomás HA, Rodrigues AF, Alves PM, et al. Lentiviral gene therapy vectors: Challenges and future directions. InTech. 2013.

38. McGinley L, McMahon J, Strappe P, Barry F, Murphy M, et al. Lentiviral vector mediated modification of mesenchymal stem cells $\&$ enhanced survival in an in vitro model of ischaemia. Stem Cell Res Ther 2011; 2: 12.

39. Chira S, Jackson CS, Oprea I, Ozturk F, Pepper MS, et al. Progresses towards safe and efficient gene therapy vectors. Oncotarget 2015; 6: 30675-30703.

40. Suzuki T, Kawamura K, Li Q, Okamoto S, Tada Y, et al. Mesenchymal stem cells are efficiently transduced with adenoviruses bearing type 35 -derived fibers and the transduced cells with the IL28A gene produces cytotoxicity to lung carcinoma cells co-cultured. BMC Cancer 2014; 14: 713.

41. Hammer K, Kazcorowski A, Liu L, Behr M, Schemmer P, et al. Engineered adenoviruses combine enhanced oncolysis with improved virus production by mesenchymal stromal carrier cells. Int J Cancer 2015; 137: 978-990.

42. Nayerossadat N, Maedeh T, Ali PA. Viral and nonviral delivery systems for gene delivery. Adv Biomed Res 2012; 1: 27.

43. Knaan-Shanzer S, van de Watering MJ, van der Velde I, Goncalves MA, Valerio D, et al. Endowing human adenovirus serotype 5 vectors with fiber domains of species B greatly enhances gene transfer into human mesenchymal stem cells. Stem Cells 2015; 23 : 1598-1607.

44. Yin T, He S, Su C, et al. Genetically modified human placentaderived mesenchymal stem cells with FGF-2 and PDGF-BB enhance neovascularization in a model of hindlimb ischemia. Molecular Medicine Reports 2015; 12: 5093-5099.

45. Kanehira M, Xin H, Hoshino K, et al. Targeted delivery of NK4 to multiple lung tumors by bone marrowderived mesenchymal stem cells. Cancer Gene Ther 2017; 14: 894-903.

46. Yang Y, Nunes FA, Berencsi K, et al. Cellular immunity to viral antigens limits E1-deleted adenoviruses for gene therapy. Proc Natl Acad Sci USA 1994; 91: 4407-4411.

47. Dahlberg J. An overview of retrovirus replication and classification. Adv Vet Sci Comp Med 1987; 32: 1-35.

48. Ke J, Zheng L, Cheung L. Orthopaedic gene therapy using recombinant adeno-associated virus vectors. Arch Oral Biol 2011; 56: 619-628.

49. Brown N, Song L, Kollu NR, Hirsch ML. Adeno-Associated Virus 
Vectors and Stem Cells: Friends or Foes? Hum Gene Ther 2017; 28: 450-463.

50. Wright JF. Transient transfection methods for clinical adenoassociated viral vector production. Hum Gene Ther 2009; 20: 698706.

51. Nayak S, Herzog RW. Progress and prospects: immune responses to viral vectors. Gene Ther 2010; 17: 295-304.

52. Naso MF, Tomkowicz B, Perry WL, Strohl WR. Adeno-associated virus (AAV) as a vector for gene therapy. BioDrugs 2017; 31: 317334.

53. Wu Z, Asokan A, Samulski RJ. Adeno-associated virus serotypes: Vector toolkit for human gene therapy. Mol Ther 2006; 14: 316-327.

54. Hirsch ML, Wolf SJ, Samulski, RJ. Delivering transgenic DNA exceeding the carrying capacity of AAV vectors. Methods Mol Biol 2016; 1382: 21-39.

55. Stender S, Murphy M, O'Brien T, Stengaard C, UlrichVinther M, Søballe K, et al. Adeno-associated viral vector transduction of human mesenchymal stem cells. Eur Cell Mater 2007; 13: 93-99.

56. Ramamoorth M, Narvekar A. Non viral vectors in gene therapy- an overview. J Clin Diagn Res 2015; 9: GE01-GE06.

57. Helledie T, Nurcombe V, Cool SM. A simple and reliable electroporation method for human bone marrow mesenchymal stem cells. Stem Cells Dev 2008; 17: 837-848.

58. Helledie T, Nurcombe V, Cool SM. A simple and reliable electroporation method for human bone marrow mesenchymal stem cells. Stem Cells Dev 2018; 17: 837-848.

59. Hardee CL, Arévalo-Soliz LM, Hornstein BD, Zechiedrich L. Advances in non-viral DNA vectors for gene therapy. Genes (Basel) 2017; 8: 211-219.

60. Boura JS, Santos FD, Gimble JM, Cardoso CM, Madeira C, Cabral JM, et al. Direct head-to-head comparison of cationic liposomemediated gene delivery to mesenchymal stem/stromal cells of different human sources: a comprehensive study. Hum Gene Ther Methods 2013; 24: 38-48.

61. Li LM, Ruan GX, HuangFu MY, Chen ZL, Liu HN, Li LX, et al. ScreenFect A: An efficient and low toxic liposome for gene delivery to mesenchymal stem cells. Int J Pharm 2015; 488: 1-11.

62. Das J, Choi YJ, Yasuda H, Han JW, Park C, Song H, et al. Efficient delivery of C/EBP beta gene into human mesenchymal stem cells via polyethylenimine-coated gold nanoparticles enhances adipogenic differentiation. Sci Rep 2016; 6: 33784.

63. Chen ZY, He CY, Ehrhardt A, Kay MA. Minicircle DNA vectors devoid of bacterial DNA result in persistent and high-level transgene expression in vivo. Mol Ther 2003; 8: 495-500.

64. Huang M, Chen Z, Hu S, Jia F, Li Z, Hoyt G, et al. Novel minicircle vector for gene therapy in murine myocardial infarction. Circulation 2019; 120: S230-S237.

65. Munye MM, Tagalakis AD, Barnes JL, Brown RE, McAnulty RJ, Howe SJ, et al. Minicircle DNA provides enhanced and prolonged transgene expression following airway gene transfer. Sci Rep 2016; 6: 23125.

66. Boura JS, Vance M, Yin W, Madeira C, Lobato da Silva C, Porada $\mathrm{CD}$, et al. Evaluation of gene delivery strategies to efficiently overexpress functional HLA-G on human bone marrow stromal cells. Mol Ther Methods Clin Dev 2014; 1: 14041.

67. Tidd N, Michelsen J, Hilbert B, Quinn JC. Minicircle Mediated Gene Delivery to Canine and Equine Mesenchymal Stem Cells. Int J Mol Sci 2017; 8(4): 819 